\title{
Chylous ascites as a complication of left sided robot-assisted laparoscopic partial nephrectomy
}

\author{
Gaurav Pahouja $^{1}$, Kalpit Patel ${ }^{1}$, Daniel J. Ricchiuti ${ }^{2}$ \\ ${ }^{1}$ Northeast Ohio Medical University, Akron General Medical Center Department of Urology, Akron, Ohio, United States; \\ 2 St. Elizabeth Health Center, Division of Urology, Youngstown, Ohio, United States.
}

\begin{abstract}
Summary Objective: The aim of the study was to present a case series of the sparsely reported complication of chylous ascites (CA) after left sided robot-assisted laparoscopic partial nephrectomy (RALPN), identify possible risk factors for the development of postoperative CA, and explore current recommendations for identification, management and prevention of $C A$.

Material and methods: A retrospective review of patients that were treated with a RALPN during a one year time period (August 2012 to August 2013) by one surgeon at our institution was conducted. A total of 12 patients were included in the study. Demographics, tumor characteristics, and perioperative outcomes were assessed.

Results: Three patients in the study experienced postoperative CA. All three patients had left sided surgery. The initial clinical suspicion for CA was raised due to complaints of abdominal pain with increased milky appearance of JP fluid. $J P$ triglycerides were elevated in all three patients. The patients responded to conservative measures, with two patients treated with medium chain triglyceride diets and one patient treated with total parenteral nutrition (TPN). Among the patients treated with RALPN, the group that was diagnosed with postoperative CA (CA group) was found to have a statistically significant lower average body mass index (BMI) as compared to the group that did not have CA (non-CA group) $\left(24.67 \mathrm{~kg} / \mathrm{m}^{2}\right.$ in the CA group versus 31.77 $\mathrm{kg} / \mathrm{m}^{2}$ in the non-CA group; $\left.P=0.026\right)$. Other demographic data, tumor characteristics, and perioperative outcomes were similar in both groups.

Conclusions: CA as a result of RALPN is a newly reported and rare postoperative complication. As utilization of RALPN continues to increase, urologists should be aware of this possible complication and be adept at diagnosing and managing CA. We suggest that left sided retroperitoneal surgery and a lower BMI preoperatively be considered risk factors for developing this complication.
\end{abstract}

KEY WORDS: Chylous ascites; Left; Robotics; Laparoscopy; Partial nephrectomy; Body mass index.

Submitted 11 April 2016; Accepted 22 May 2016

\section{INTRODUCTION}

The incidence of renal cell carcinoma in the United States is estimated to be approximately 15.6 per 100,000 men and women per year (1-2). The lifetime risk of developing renal cell carcinoma is estimated to be $1.6 \%$ (1-2). Renal cell and renal pelvis cancer is fairly common compared to many other types of cancer, ranking as the 9th most common type of cancer in the United States and comprising approximately 3.7\% of all new cancer cases in the United States (1-2). The incidence of renal cell carcinoma has been rising on average $1.4 \%$ each year over the past 10 years while death rates have been falling on average of $0.7 \%$ each year (1). Current surgical management values favorable oncologic outcomes paired with a nephron sparing approach. Partial nephrectomy is emerging as a minimally invasive nephron sparing approach for renal cancers, with the options of an open, laparoscopic, or robotic approach (3-4). Robotic partial nephrectomy is gaining popularity secondary to acceptable safety and outcomes (5-6). Advantages of the robotic approach to partial nephrectomy as compared to the open approach include decreased blood loss, decreased pain, shorter length of hospital stay, improved cosmesis, and more rapid convalescence (7-8). As the robotic approach to partial nephrectomy continues to gain support, possible complications must be explored.

Chylous ascites (CA), also known as chyloperitoneum, is a rare complication of retroperitoneal surgery that can often go undiagnosed and untreated (9-11). CA is an accumulation of lymphatic fluid within the peritoneal cavity. In the surgical setting, it is hypothesized that disruption of the retroperitoneal lymph vessels leads to lymphoperitoneal fistula formation (12-13). Analysis of peritoneal fluid is diagnostic and will produce a milky, sterile, odorless fluid with an alkaline $\mathrm{pH}$, total protein $>3 \mathrm{~g} / \mathrm{dL}$, total fat between 0.4 and $4.0 \mathrm{~g} / \mathrm{dL}$ and predominance of lymphocytes on the differential white blood cell count (14). Historically, CA was most commonly reported as a complication of abdominal aortic surgery. CA has been reported in the literature after urologic surgeries including retroperitoneal lymphadenectomy for testis and renal cancers, Wilm's tumor resection, and laparoscopic radical and donor nephrectomy (15-31). Chylous leakage and ascites has only been reported in very rare instances in operative techniques and surgical outcomes in robot-assisted laparoscopic partial nephrectomy (RALPN) (32-34). Our objective is to present a case series of this rare complication after left sided RALPN, identify possible risk factors for the development of postoperative CA, and explore current recommendations for identification, management, and prevention of CA. 


\section{Material AND MEthods}

\section{Study population}

A retrospective review of patients that were treated with a RALPN during a one year time period (August 2012 to August 2013) by one surgeon at our institution was conducted. The medical records of these patients were gathered and analyzed. Data collected included diagnosis of postoperative CA, demographics, tumor characteristics, and perioperative outcomes. Inclusion criteria included the presence of a renal mass or lesion, as confirmed by radiologic imaging, and subsequent RALPN performed by the same surgeon.

\section{Surgical technique}

Standard approach to left sided robot-assisted partial nephrectomy was performed (35-36). All operations were performed using the da Vinci Surgical System through a retroperitoneal approach. Briefly, the aorta is identified and the left renal artery is dissected at the level of the takeoff from the aorta. Any lymphatic channels identified are secured with surgical clips. Once the hilum is fully dissected, the renal artery is clamped. In some cases, the renal vein may also be clamped. Tumor excision and renorrhaphy are then performed.

\section{Statistical analysis}

For descriptive statistics, data is presented as mean with standard deviations. Categorical variables are presented as the percentage of total patients and the total number of patients. The between group comparisons were done utilizing two-tailed t-test or Fisher's exact test with significance defined as $\mathrm{P}<0.05$.

\section{RESULTS \\ Chylous ascites - Case series}

\section{Patient 1}

A 46-year-old man with a past medical history of hypertension and gastroesophageal reflux disease and past surgical history of hernia repair was found to have a complex cystic lesion. He also had a 20 pack-year smoking history. His preoperative computed tomography (CT) scan revealed inflammatory changes around the kidney suggestive of a soft tissue mass or complex cyst. Subsequently, he underwent a left sided RALPN. Intraoperatively, the patient was noted to have two renal arteries and one renal vein. The lesion was identified and removed from Gerota's fascia. A Jackson Pratt (JP) drain was placed and monitored postoperatively. His pathology revealed a $7 \mathrm{~cm}$ by 6 $\mathrm{cm}$ by $4.5 \mathrm{~cm}$ benign cortical cyst. On postoperative day (POD) 1, the patient complained of epigastric, left upper quadrant (LUQ), and left lower quadrant (LLQ) abdominal tenderness but has no evidence of abdominal distention. His vital signs were within normal limits at the time. In addition, the patient had high output drainage (302 $\mathrm{mL}$ ) of pink tinged, milky white fluid. Evaluation of the drain fluid revealed that the drain fluid and serum creatinine were equivalent. In addition, JP drain fluid lipase and amylase were within normal limits. However, JP drain fluid triglycerides were elevated (> 1,100 mg/dL) and the patient was subsequently diagnosed with CA. The patient was treated with total parenteral nutrition (TPN). On POD 3, JP drain fluid triglycerides decreased to $212 \mathrm{~mL}$. Besides complaints of LUQ and LLQ abdominal pain, the patient remained clinically stable and his abdominal exam was unremarkable with normal bowel sounds throughout the hospital stay. By POD 4, the drainage had decreased considerably ( $<50 \mathrm{~mL}$ day). After his diet was advanced without increased JP drainage, the JP drain was removed and he was discharged home on POD 7 with resolution of his symptoms.

\section{Patient 2}

A 56-year-old male with a past medical history of asthma and gastrointestinal reflux disease and no past surgical history presented with a left sided renal lesion. His CT scan revealed an enhancing lesion on the lower pole of his left kidney suggestive of renal cell carcinoma or angiomyolipoma and also showed perihilar lymphadenopathy. He underwent a left sided RALPN and periaortic lymph node resection for the enhancing lesion and lymphadenopathy. The patient was noted to have a solitary renal artery and solitary renal vein. A $1.5 \mathrm{~cm}$ by $1.5 \mathrm{~cm}$ by $1.2 \mathrm{~cm}$ tumor was identified and successfully removed. Pathology revealed a grade 3 clear cell renal cell carcinoma (RCC). In addition, six periaortic lymph nodes removed during surgery were negative for malignancy. On POD 1, the patient complained of left sided flank pain and mild abdominal distension was noted. His vital signs were within normal limits. By POD 2, a low volume milky leakage was identified in the JP drain. JP drain fluid evaluation revealed an elevated triglyceride count (> $1100 \mathrm{mg} / \mathrm{dL})$, with normal amylase, lipase, and creatinine levels. He was diagnosed with $\mathrm{CA}$, and started on a low fat medium chain triglyceride diet. On POD 3, he was discharged home with his JP drain in place on a low fat medium chain triglyceride diet. After two weeks, his JP drain output had decreased considerably, and his JP drain was removed as an outpatient without any further issues.

\section{Patient 3}

A 54-year-old male with a past medical history of degenerative disc disease and chronic back pain and past surgical history of hernia repair, appendectomy, left elbow surgery, right hand surgery, and right foot surgery presented for evaluation of a left renal mass. He also had a 75 pack-year smoking history. His preoperative CT scan revealed a posterior superior $3 \mathrm{~cm}$ left renal mass. He was treated with a left sided RALPN. Intraoperatively, he had a large amount of lymphatic vessels that needed to be transected to access the renal artery which were cauterized as best as possible. He was noted to have a solitary renal artery and vein. His tumor was identified, removed, and sent for pathological evaluation which revealed a $3 \mathrm{~cm}$ by $3 \mathrm{~cm}$ by $2.5 \mathrm{~cm}$ tumor composed of clear cell RCC. On POD 3, his JP drain was draining a pink tinged, milky fluid.

Laboratory analysis of the JP drain fluid showed normal JP drain fluid creatinine, lipase, and amylase but elevated JP drain fluid triglycerides (> 1,100 mg/dL). CA was identified, and the patient was started on a medium chain fatty acid diet. By POD 5, he was doing well clini- 
cally but persisted to have elevated drain output. He was discharged home on POD 5 with his JP drain in place. The patient returned 7 days after discharge (POD 12) with a chills, fatigue, cough, and abdominal pain. His vital signs revealed a blood pressure of $124 / 70 \mathrm{mmHg}$, respiratory rate of 16 , tachycardia with a heart rate of 124 beats per minute, and fever of $38.44^{\circ} \mathrm{C}$. His abdomen was moderately tender in the LLQ and was mildly distended. In addition, his JP drain was in place and draining minimal serous cream tinged fluid. His white blood cell count was elevated $\left(>12,000 \mathrm{~mm}^{3}\right)$. The patient had a CT scan which was negative for a pelvic abscess. A Gram stain of the JP drain fluid revealed group A streptococcus pyogenes and severe sepsis was identified. He was treated with fluid resuscitation and broad spectrum antibiotics. In addition, he was placed on TPN. The patient improved clinically and was started on a clear liquid diet on POD 16. On POD 17 , his TPN was weaned and he was started on a low fat medium chain fatty acid diet.

On POD 18, the JP drain output decreased to $10 \mathrm{~mL}$ over 24 hours and the JP drain was removed. The patient was again discharged in stable condition on POD 20 without any further complications.

\section{Demographics}

A total of 12 patients had a RALPN by one surgeon during a one year long time period and were included in the study. Three of the patients in the study experienced postoperative CA. All three patients had underwent a left sided RALPN. Among the patients treated with RALPN, the group that was diagnosed with postoperative CA (CA group) was found to have a statistically significant lower average body mass index (BMI) as compared to the group that did not have CA (non-CA group) $\left(24.67 \mathrm{~kg} / \mathrm{m}^{2}\right.$ in the CA group versus $31.77 \mathrm{~kg} / \mathrm{m}^{2}$ in the non-CA group; $\mathrm{P}=$ 0.026). The $C A$ and non-CA groups were comparable in terms of other demographic data (Table 1).

Table 1.

Demographic data and intraoperative findings of patient in Center A and Center B included in this study.

\begin{tabular}{|lccc|}
\hline Variable & $\begin{array}{c}\text { CA after RALPN } \\
(\mathbf{n}=\mathbf{3})\end{array}$ & $\begin{array}{c}\text { No CA after RALPN } \\
(\mathbf{n}=\mathbf{9})\end{array}$ & P value* \\
\hline Age (years) & $51.67(5.86)$ & $53.44(9.44)$ & 0.77 \\
\hline BMI (kg/m²) & $24.67(2.43)$ & $31.77(4.39)$ & $\mathbf{0 . 0 2 6}$ \\
\hline Males (\%) & $100(3)$ & $55.56(5)$ & 0.49 \\
\hline Caucasian (\%) & $100(3)$ & $100(9)$ & 1 \\
\hline Prior abdominal surgery (\%) & $66.67(2)$ & $66.67(6)$ & 1 \\
\hline Left sided surgery (\%) & $100(3)$ & $55.56(5)$ & 0.49 \\
\hline
\end{tabular}

RALPN = robot-assisted laparoscopic partial nephrectomy; $C A=$ chylous ascites; $B M I=$ body mass index.

Data presented as mean, with standard deviation in parenthesis, for age and BMI.

Data presented as percentage of patients, with number of patients in parenthesis, for males, Caucasian, prior abdominal surgery, and left sided surgery.

* Group comparison $P$ value performed using two-tailed t-test for age and $B M I$ and Fisher's exact test for males, Caucasian, prior abdominal surgery, and left sided surgery with statistical significance set to $P<0.05$.
Table 2.

Tumor characteristics of RALPN patients CA group versus non-CA group.

\begin{tabular}{|lccc|}
\hline Variable & $\begin{array}{c}\text { CA after RALPN } \\
\text { ( } \mathbf{=} \mathbf{3})\end{array}$ & $\begin{array}{c}\text { No CA after RALPN } \\
\text { (n = 9) }\end{array}$ & P value* \\
\hline Tumor size (cm) & $3.83(2.84)$ & $4.07(1.71)$ & 0.86 \\
\hline Tumor location (\%) & $33.33(1)$ & $44.44(4)$ & 1 \\
\hline Upper pole & $0(0)$ & $11.11(1)$ & 1 \\
\hline Middle pole & $33.33(1)$ & $33.33(3)$ & 1 \\
\hline Lower pole & $33.33(1)$ & $0(0)$ & 0.25 \\
\hline Gerota's fascia & $0(0)$ & $11.11(1)$ & 1 \\
\hline Perihilar & & & 1 \\
\hline Pathologic findings (\%) & $66.67(2)$ & $77.78(7)$ & 1 \\
\hline Clear cell RCC & $0(0)$ & $11.11(1)$ & 1 \\
\hline Chromophobe RCC & $33.33(1)$ & $0(0)$ & 0.25 \\
\hline Benign cortical cyst & $0(0)$ & $11.11(1)$ & 1 \\
\hline Angiomyolipoma & & & \\
\hline
\end{tabular}

RALPN = robot-assisted laparoscopic partial nephrectomy;

$C A=$ chylous ascites; $R C C=$ renal cell carcinoma.

Data presented as mean, with standard deviation in parenthesis, for tumor size. Data presented as percentage of patients, with number of patients in parenthesis, for tumor location and pathologic findings.

* Group comparison P value performed using two-tailed t-test for tumor size and Fisher's exact test for tumor location and pathologic findings with statistical significance set to $P<0.05$.

Perioperative outcomes

When the CA group is compared to the non-CA group, there were no significant differences in tumor characteristics including tumor size, tumor location, and final pathologic findings (Table 2).

In terms of perioperative outcomes, the CA group was comparable to the non-CA group (Table 3). There were no statistically significant differences in operative duration, estimated blood loss, and variations in renal anatomy.

Table 3.

Perioperative outcomes of RALPN patients $C A$ group versus non-CA group.

\begin{tabular}{|lccc|}
\hline Variable & $\begin{array}{c}\text { CA after RALPN } \\
\text { ( } \mathbf{n = 3 )}\end{array}$ & $\begin{array}{c}\text { No CA after RALPN } \\
\text { (n = 9) }\end{array}$ & P value* \\
\hline Operative duration (min) & $249.67(12.74)$ & $279.78(43.73)$ & 0.28 \\
\hline Estimated blood loss (mL) & $333.33(236.29)$ & $500(446.51)$ & 0.56 \\
\hline $\begin{array}{l}\text { Greater than 2 renal } \\
\text { vessels on side of surgery (\%) }\end{array}$ & $33.33(1)$ & $44.44(4)$ & 1 \\
\hline $\begin{array}{l}2 \text { or more renal arteries } \\
\text { on side of surgery (\%) }\end{array}$ & $33.33(1)$ & $22.22(2)$ & 1 \\
\hline $\begin{array}{l}2 \text { or more renal veins } \\
\text { on side of surgery (\%) }\end{array}$ & $0(0)$ & $22.22(2)$ & 1 \\
\hline
\end{tabular}

$R A L P N=$ robot-assisted laparoscopic partial nephrectomy;

$\mathrm{CA}=$ chylous ascites.

Data presented as mean, with standard deviation in parenthesis, for operative duration and estimated blood loss. Data presented as

percentage of patients, with number of patients in parenthesis, for greater than 2 renal vessels on side of surgery, 2 or more renal arteries on side of surgery, and 2 or more renal veins on side of surgery.

* Group comparison P value performed using two-tailed t-test and Fisher's exact test with statistical significance set to $P<0.05$. 


\section{Discussion}

Chylous fluid accumulation has been reported to arise from disruption of the lymphatic system secondary to traumatic injury or obstruction (37). The lymphatic system consists of a linear network of lymphatic vessels and lymphoid organs. Lymph, a collection of tissue fluids, cells and extracellular molecules, are drained into lymphatic capillaries and then into lymphatic vessels, lymph nodes, and lymph trunks and then returned to the blood circulation (38-39). Classically, the renal lymphatic system follows along a similar pathway as the renal venous drainage. Renal lymphatics coalesce to form lymphatic trunks that exit the renal hilum. The right renal lymphatic trunks drain to the paracaval, precaval, retrocaval, and interaortocaval lymph nodes which extend from the diaphragm to the right common iliac artery (40-41). The left renal lymphatic drainage is to the paraaortic, preaortic, and retroaortic lymph nodes which extend from the diaphragm to the inferior mesenteric artery (40-41). Most of the efferent vessels from these groups of lymph nodes then converge to form the right and left lumbar trunks which subsequently drain to the cisterna chyli (40-41). It is important for urologists performing RALPN to be aware of the clinical presentation, diagnosis, and treatment of CA, as CA may lead to serious complications. The most common clinical manifestations of CA are abdominal distention and nonspecific pain (42). Additional findings may include pleural effusions, lower extremity edema, dyspnea, cachexia, malnutrition, lymphadenopathy, hernia, fevers, and night sweats $(9,37,42-43)$. CA results in a loss of chyle to the peritoneal cavity which can progress to nutritional deficiency with loss of crucial electrolytes, proteins, immunoglobulins, lymphocytes, vitamins, lipids, and water (44). Additionally, the continued loss of lymphocyte-rich lymph to the peritoneal cavity paired with loss of protein can cause hypogammaglobulinemia and a resultant immunodeficiency with increased susceptibility to infection (45). Finally, the bioavailability of drugs can be severely deteriorated with CA (46-47). In the postoperative setting, these losses can lead to an increased morbidity and mortality rate (48).

$\mathrm{CA}$ is routinely diagnosed using peritoneocentesis. On analysis, the triglyceride level in the milky appearing ascitic fluid sample will be elevated - the standard is greater than $110 \mathrm{mg} / \mathrm{dL}$ (37). Rather than using an absolute level, the ratio of ascitic triglyceride to plasma triglyceride can be used as well. A ratio between 2:1 and $8: 1$ is associated with CA (43). To further confirm suspicion, lymphoscintigraphy, lymphography with CT, abdominal ultrasound, as well as magnetic resonance imaging can be utilized to explore the possibility of a chyloperitoneum.

Most of the treatment for CA is supportive in nature. Nutritional support is essential to replenish vitamin, electrolyte, and fluid deficiencies. To achieve symptomatic relief, the fluid must be removed via diuretics, elevation of legs, supportive stockings, dietary changes, and also repeated paracentesis (37). Since chyle is made up of long-chain fatty acids, minimizing high fat foods paired with medium chain triglyceride supplementation may decrease chyle development (49).

In cases of resistant or postsurgical CA, a more vigorous treatment regimen of bowel rest and TPN may be warranted (50). Somatostatin therapy in addition to TPN has also proven beneficial in several instances in decreasing chyloperitoneum and alleviating symptoms (50-51). In cases where the CA continues despite adequate mechanical and pharmacological therapy, there are several possible surgical options. If the leak is discovered early, then it can be managed with staples or clips (52). Another possibility is peritoneovenous shunting in order to prevent nutritional depletion (53). Some success has also been seen with using fibrin glue applications with and without mesh after careful dissection of the leakage area $(52,54)$. As CA remains a rare complication, there is yet to be a standard surgical approach.

It has been identified in the literature that during laparoscopic donor nephrectomy (LDN), the incidence of CA increases when attempting to preserve the length of the renal artery and vein. In order to do so, a more extensive dissection of the renal hilum is necessary. In LDN, there has been a higher incidence of CA following the removal of the left donor kidney (22-24). The higher incidence of CA associated with left sided LDN versus the right has been attributed to the lymphatic anatomy as well as the higher frequency of extraction of the left kidney, as the left kidney is anatomically preferred for transplantation. The ascending vertical lymphatic channels parallel the aorta into the retroperitoneum and merge posterior and medial to the aorta forming the cisterna chyli. This saclike dilatation of the ascending vertical lymphatic channels is located anterior to the first and second lumbar vertebrae (22). However, there are many anatomical variants of the lymphatic channels (14).

The location of the cisterna chyli is thought to be the anatomic cause of the increased incidence of CA following left sided LDN. Of note, CA does not appear to be a complication of open nephrectomy. This finding is attributed to the pneumoperitoneum which decreases the leakage of the low pressure lymph vessels. On the contrary, in the absence of pneumoperitoneum during an open surgery, these lymphatics are more readily identified and ligated (34).

CA as a result of partial nephrectomy is a rare postoperative complication that has been sparsely reported. Weiser et al. reported one incidence of CA after an open partial nephrectomy for Wilms tumor (30). Wan et al. reported five patients out of 39 experiencing CA after upper-pole heminephrectomy for duplex kidney (31). Even fewer reports of CA after RALPN have been published.

Tanagho et al. reported one case of CA out of 886 patients treated with robotic-assisted partial nephrectomy for a renal mass (32). Hillyer et al. reported one case of CA out of nine patients that underwent bilateral robot-assisted partial nephrectomy for renal masses (33). In both instances, the side of the CA was not specified.

In reviewing the patients, this study found that three out of 12 patients that had a RALPN by a single surgeon over the span of a year had the postoperative complication of CA. All three patients had their procedures done on the left side. This study found that BMI was a statistically significant variable between patients that had CA and those that did not. This may suggest that patients with a lower 
BMI have a higher risk of developing CA after a RALPN, and those with a higher BMI have a lower risk of developing CA after a RALPN. Finally, the patient series of this study paired with the reports in the literature discussed may suggest that RALPN, especially on the left side, is a risk factor for developing the complication of postoperative CA.

Urologists should be well aware of techniques to prevent CA during a RALPN. As mentioned, an extensive renal hilar dissection during robotic surgery may increase the risk of CA, especially on the left side. The length and position of the left kidney make it necessary to perform a dissection close to the aorta. As described, many lymph vessels and major lymph trunks are concentrated along the paraaortic region. Therefore, during dissection of the renal vessels, care should be taken to identify and avoid, if possible, the paraaortic lymphatic channels. In addition, caution should be utilized when identifying and dissecting any possible accessory renal vessels. Over dissection while trying to locate such accessory vessels may result in higher risk of postoperative CA. Of note, with RALPN and pure laparoscopic cases, monopolor and bipolar coagulation are often used to perform dissections and achieve lymphostasis. Therefore, it is critical to be aware of lymphatics and the cisterna chyli that lie in close proximity to the aorta during left RALPN and utilize laparoscopic clipping of major lymphatics around the perihilar and retroperitoneal tissue during renal pedical dissection. This technique may help prospectively prevent CA.

Limitations of this study should be discussed. The limitations of the study included the retrospective nature and small sample size which partially limit the significance of the outcome assessments. A multivariate analysis may validate the findings of this study. However, the sample size is small. In addition, aside from the possible risk factors of left sided surgery and BMI, the study was unable to identify the exact risk factors and etiology of CA following RALPN. Large, prospective, randomized studies are still needed to better study the complication of CA following RALPN and definitively characterize the possible risk factors and etiologies. Nevertheless, this study was able to identify the postoperative complication of CA after RALPN which has not been widely reported in the literature.

\section{Conclusions}

CA as a result of RALPN is a newly reported and rare postoperative complication. As utilization of RALPN continues to increase, urologists performing the case should be aware of this possible complication and be adept at diagnosing and managing CA. A patient with a lower BMI preoperatively may be at a higher risk of developing postoperative CA. In addition, patients undergoing left sided RALPN may have a higher risk of developing postoperative CA. If there is increased drainage or milky discharge postoperatively, the drain fluid should be sent for triglycerides and other appropriate laboratory evaluation. If drainage is consistent with lymph fluid, dietary changes should be implemented with a low fat medium chain triglyceride diet and/or TPN. Once drain output decreases, the drain may be removed. For severe or refractory cases, surgical intervention may be required. We suggest that left sided retroperitoneal surgery and a lower BMI preoperatively be considered risk factors for developing postoperative CA, and we emphasize the importance of urologists being able to identify and appropriately treat this potentially unforeseeable outcome.

\section{References}

1. Howlader N, Noone AM, Krapcho M, et al. SEER cancer statistics review, 1975-2012, based on november 2014 SEER data submission. National Cancer Institute. 2015.

2. Chow W, Dong LM, Devesa SS. Epidemiology and risk factors for kidney cancer. Nat Rev Urol. 2010; 7:245-247.

3. Uzzo RG, Novick AC. Nephron sparing surgery for renal tumors: indications, techniques and outcomes. J Urol. 2001; 166:6-18.

4. Fergany AF, Hafes KS, Novick AC. Long-term results of nephron sparing surgery for localized renal cell carcinoma: 10-year followup. J Urol. 2000; 163:442-445.

5. Caruso RP, Phillips CK, Kau E, Taneja SS, Stifelman MD. Robot assisted laparoscopic partial nephrectomy: initial experience. J Urol. 2006; 176:36-39.

6. Zhang X, Yan J, Ren Y, et al. Robot-assisted versus laparoscopic partial nephrectomy for localized renal tumors: a meta-analysis. Int J Clin Exp Med. 2014; 7:4770-4779.

7. Mottrie A, De Naeyer G, Schatteman P, et al. Impact of the learning curve on perioperative outcomes in patients who underwent robotic partial nephrectomy for parenchymal renal tumours. Eur Urol. 2010; 58:127-132.

8. Deane LA, Lee HJ, Box GN, et al. Robotic versus standard laparoscopic partial/wedge nephrectomy: a comparison of intraoperative and perioperative results from a single institution. J Endourol. 2008; 22:947-952.

9. Browse NL, Wilson NM, Russo F, et al. Aetiology and treatment of chylous ascites. Br J Surg. 1992; 79:1145-1150.

10. Shapiro AM, Bain VG, Sigalet DL, Kneteman NM. Rapid resolution of chylous ascites after liver transplantation using somatostatin analog and total parenteral nutrition. Transplantation. 1996; 61:1410-1411.

11. Besson R, Gottrand F, Saulnier P, Giard H, Debeugny P. Traumatic chylous ascites: conservative management. J Pediatr Surg. 1992; 27:1543.

12. Baniel J, Foster RS, Rowland RG, et al. Management of chylous ascites after retroperitoneal lymph node dissection for testicular cancer. J Urol. 1993; 150:1422-1444.

13. Leibovitch I, Mor Y, Golomb J, Ramon J. The diagnosis and management of postoperative chylous ascites. J Urol. 2002; 167:449-457.

14. Pabst TS 3rd, McIntyre KE Jr, Schilling JD, et al. Management of chyloperitoneum after abdominal aortic surgery. Am J Surg. 1993; 166:198-199.

15. Allen W, Parrott TS, Saripkin L, Allan C. Chylous ascites following retroperitoneal lymphadenectomy for granulos a cell tumor of the testis. J Urol. 1986; 135:797-798.

16. Dharman K, Temes SP, Wetherell FE, Kendrick MJ. Chyloperitoneum and chylothorax: a combined rare occurrence after retroperitoneal lymphadenectomy and radiotherapy for testis tumor. J Urol. 1984; 131: 346-347.

17. Selli C, Carini M, Mottola A, Barbagli G. Chylous ascites after 
retroperitoneal lymphadenectomy: successful management with peritoneovenous shunt. Urol Int. 1984; 39:58-60.

18. Ferrigni RG, Novicki DE. Chylous ascites complicating genitourinary oncological surgery. J Urol. 1985; 134:774-776.

19. Halachmi S, Noyman A, Moskovitz B, Nativ O. Successful treatment of postretroperitoneal lymph node dissection in massive chylous ascites. Eur Urol. 1995; 28:119-121.

20. Weston PM, Greenland JE, Wallace DM. Chylous ascites following retroperitoneal lymph node dissection for testis cancer. $\mathrm{Br} J$ Urol. 1992; 70:688-689.

21. Jayabose S, Kogan S, Berezin S, et al. Combined occurrence of chyloperitoneum and chylothorax after surgery and chemotherapy for Wilms' tumor. Cancer. 1989; 64:1790-1795.

22. Kim BS, Yoo ES, Kim TH, Kwon TG. Chylous ascites as a complication of laparoscopic nephrectomy. J Urol. 2010; 184:570-574.

23. Meulen ST, van Donselaar-van der Pant KA, Bemelman FJ, Idu MM. Chylous ascites after laparoscopic hand-assisted donor nephrectomy: is it specific for the left-side?. Urol Ann. 2013; 5:45-46.

24. Tiong HY, Goel RK, White WM, et al. Chylous ascites after laparoscopic donor nephrectomy. Asian J Endosc Surg. 2005; 8:34-39.

25. Capocasale E, Iaria M, Vistoli F, et al. Incidence, diagnosis, and treatment of chylous leakage after laparoscopic live donor nephrectomy. Transplantation. 2012; 93:82-86.

26. Aerts J, Matas A, Sutherland D, Kandaswamy R. Chylous ascites requiring surgical intervention after donor nephrectomy: case series and single center experience. Am J Transplant. 2010; 10:124-128.

27. Sinha A, Mamode N. Laparoscopic management of chylous ascites following laparoscopic donor nephrectomy. BMJ Case Rep. 2010; 2010: bcr0820092141.

28. Shah SS, Ahmed K, Smith R, et al. Chylous ascites following radical nephrectomy: a case report. J Med Case Rep. 2008; 2:3.

29. Breda A, Veale J, Liao J, Schulam PG. Complications of laparoscopic living donor nephrectomy and their management: the UCLA experience. Urology. 2007; 69:49-52.

30. Weiser AC, Lindgren BW, Ritchey ML, Franco I. Chylous ascites following surgical treatment for wilms tumor. J Urol. 2003; 170:1667-1669.

31. Wan Y, Tang Z, Chen Z, et al. Chylous leakage after retroperitoneoscopic upper-pole heminephrectomy for duplex kidney. Zhong Nan Da Xue Xue Bao Yi Xue Ban. 2012; 37:405-407.

32. Tanagho YS, Kaouk JH, Allaf ME, et al. Perioperative complications of robot-assisted partial nephrectomy: analysis of 886 patients at 5 united states centers. Urology. 2013; 81:573-579.

33. Hillyer SP, Autorino R, Layder H, et al. Robotic versus laparoscopic partial nephrectomy for bilateral synchronous kidney tumors: single-institution comparative analysis. Urology. 2011; 78:808-812.

34. Ho H, Schwenter C, Neururer R, et al. Robotic-assisted laparoscopic partial nephrectomy: surgical technique and clinical outcomes at 1 year. BJU Int. 2009; 103:663-668.

35. Rogers CG, Singh A, Blatt AM, et al. Robotic partial nephrectomy for complex renal tumors: surgical technique. Eur Urol. 2008; 53:514-521.

36. Benway BM, Bhayani SB. Robot-assisted partial nephrectomy: evolution and recent advances. Curr Opin Urol. 2010; 20:119-124.

37. Press OW, Press NO, Kaufman SD. Evaluation and management of chylous ascites. Ann Intern Med. 1982; 96:358-364.
38. Choi I, Lee S, Hong YK. The new era of the lymphatic system: no longer secondary to the blood vascular system. Cold Spring Harb Prospect Med. 2012; 2:a006445.

39. Alitalo K, Carmeliet P. Molecular mechanisms of lymphangiogenesis in health and disease. Cancer Cell. 2002; 1:219-227.

40. Wein AJ, Kavoussi LR, Novick AC, et al. Campbell-walsh urology. $11^{\text {th }}$ ed. Philadelphia: Elsevier, 2015.

41. Smith Jr JA, Howards SS, Preminger GM. Human's atlas of urologic surgery: expert consult. $3^{\text {rd }}$ Ed. Philadelphia: Saunders, 2012.

42. Steinemann DC, Dindo D, Clavien PA, Nocito A. Atraumatic chylous ascites: systematic review on symptoms and causes. J Am Coll Surg. 2011; 212:899-905.

43. Aalami OO, Allen DB, Organ CH Jr. Chylous ascites: a collective review. Surgery. 2000; 128:761-778.

44. Zilversmit $D B$. The composition and structure of lymph chylomicrons in dog, rat, and man. J Clin Invest. 1965; 44:1610-1622.

45. Camiel MR, Benninghoff DL, Alexander LL. Chylous effusions, extravasation of lymphographic contrast material, hypoplasia of lymph nodes and lymphocytopenia. Chest. 1971; 59:107-110.

46. Taylor MD, Kim SS, Vaias LJ. Therapeutic digoxin level in chylous drainage with no detectable plasma digoxin level. Chest. 1998; 114:1482-1484.

47. Strange C, Nicolau DP, Dryzer SR. Chylous transport of amiodarone. Chest. 1992; 101:573-574.

48. Gaglio PJ, Leevy CB, Koneru B. Peri-operative chylous ascites. J Med. 1996; 27:369-376.

49. Weinstein LD, Scanlon GT, Hersh T. Chylous ascites. Management with medium-chain triglycerides and exacerbation by lymphangiography. Am J Dig Dis. 1969; 14:500-509.

50. Ijichi H, Soejima Y, Taketomi A, et al. Successful management of chylous ascites after living donor liver transplantation with somatostatin. Liver Int. 2008; 28:143-145.

51. Baran M, Cakir M, Yüksekkaya HA, et al. Chylous ascites after living related liver transplantation treated with somatostatin analog and parenteral nutrition. Transplant Proc. 2008; 40:320-321.

52. Jensen EH, Weiss CA $3^{\text {rd }}$. Management of chylous ascites after laparoscopic cholecystectomy using minimally invasive techniques: a case report and literature review. Am Surg. 2006; 72:60-63.

53. Matsufuji $H$, Nishio $T$, Hosoya $R$. Successful treatment for intractable chylous ascites in a child using a peritoneovenous shunt. Pediatr Surg Int. 2006; 22:471-473.

54. Zeidan S, Delarue A, Rome A, Roquelaure B. Fibrin glue application in the management of refractory chylous ascites in children. $J$ Pediatr Gastroenterol Nutr. 2008; 46:478-481.

\section{Correspondence}

Gaurav Pahouja, MD (Corresponding Author)

gpahouja@neomed.edu

Kalpit Patel, MD

kalpatel428@gmail.com

Northeast Ohio Medical University,

Akron General Medical Center Department of Urology,

Akron, Ohio, United States

Daniel J. Ricchiuti, MD

dricchiuti@yahoo.com

St. Elizabeth Health Center, Division of Urology, Youngstown,

Ohio, United States 